\title{
P68 - Immunogenicity of 13-valent conjugate pneumococcal vaccine (PCV13) in children with asthma or recurrent wheeze receiving inhaled corticosteroids (ICS)
}

\author{
Lefki Giannopoulou ${ }^{1 *}$, Polytimi Panaghiotopoulou-Gartagani ${ }^{2}$, Eleni Kyritsi ${ }^{3}$, Athanasios Kaditis ${ }^{2}$, \\ Maria Theodoridou', Vasiliki Spoulou ${ }^{4}$
}

From 3rd Pediatric Allergy and Asthma Meeting (PAAM)

Athens, Greece. 17-19 October 2013

\section{Introduction}

Patients with asthma have been reported to be at increased risk of invasive pneumococcal disease (IPD). Therefore, routine vaccination against Streptococcus Pneumoniae is suggested for all children suffering from asthma or recurrent wheeze. Inhaled corticosteroids (ICS) are the preferred treatment for initiating longterm control therapy in these children. Their effect on the immunogenicity of PCV13 is yet unknown.

\section{Aim}

To evaluate the immunogenicity of PCV13, in children with asthma or recurrent wheeze, receiving medium doses of ICS for extended periods of time.

\section{Method}

40 children ( 26 boys) with asthma or recurrent wheeze (mean age 4,3 $\pm 1,05$ years) were assigned to 3 groups according to the duration of their ICS therapy (1st $<30$ days, 2 nd 30-90 days, 3rd $>90$ days, dosage 200$250 \mu \mathrm{g} /$ day of inhaled fluticasone in all groups). 13 healthy age-matched children were included in the study as controls (group 0). All children received for the first time one dose of PCV13. Pneumococcal serotype (PS) specific IgG antibodies to serotypes 3 and 19A were measured by ELISA, before and 31,9 $\pm 5,6$ days after vaccination. IgG concentration $\geq 0,35 \mu \mathrm{g} / \mathrm{ml}$ was considered protective for invasive disease. For the statistical analysis SPSS17 and non-parametric tests were used. The statistical significance level was set at $\mathrm{p}<0,05$.

\section{Results}

One month after vaccination, $96,2 \%$ of all 53 children had protective IgG concentration $\geq 0,35 \mu \mathrm{g} / \mathrm{ml}$, for each serotype. The geometric mean concentration (GMC) of IgG antibodies after vaccination, the increase in the GMC and the percentage of children that achieved protective IgG concentration $\geq 0,35 \mu \mathrm{g} / \mathrm{ml}$, did not differ for neither of the serotypes, when compared among our 4 groups $(p>0,05)$.

\section{Conclusion}

The immunogenicity of PCV13, in children with asthma or recurrent wheeze, is not affected by the use of medium doses of ICS as control therapy, even after extended periods of time.

\section{Authors' details \\ ${ }^{1}$ Pediatrics Department, Tzaneio General Hospital, Piraeus, Greece. ${ }^{2}$ Pediatric Pulmonology Unit, $1^{\text {st }}$ Department of Pediatrics, National Kapodistrian University of Athens School of Medicine, "Aghia Sophia" Children's Hospital, Athens, Greece. ${ }^{3}$ Technological Institution of Athens, $1^{\text {st }}$ Nursing Department, Athens, Greece. ${ }^{4} 1^{\text {st }}$ Department of Pediatrics, National and Kapodistrian University of Athens School of Medicine, "Aghia Sophia" Children's Hospital, Athens, Greece.}

Published: 28 February 2014

doi:10.1186/2045-7022-4-S1-P123

Cite this article as: Giannopoulou et al:: P68 - Immunogenicity of 13valent conjugate pneumococcal vaccine (PCV13) in children with asthma or recurrent wheeze receiving inhaled corticosteroids (ICS). Clinical and Translational Allergy 2014 4(Suppl 1):P123.

${ }^{1}$ Pediatrics Department, Tzaneio General Hospital, Piraeus, Greece

Full list of author information is available at the end of the article 